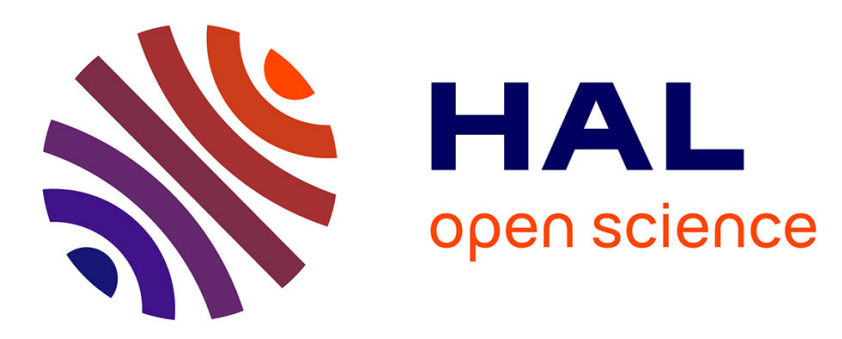

\title{
Multi-scale surface characterization in additive manufacturing using CT
}

Yann Quinsat, Claire Lartigue, Christopher Brown, Lamine Hattali

\section{To cite this version:}

Yann Quinsat, Claire Lartigue, Christopher Brown, Lamine Hattali. Multi-scale surface characterization in additive manufacturing using CT. International Joint Conference on Mechanics, Design Engineering \& Advanced Manufacturing (JCM 2016), Sep 2016, Catane, Italy. 10.1007/978-3-31945781-9_28. hal-01358916

\section{HAL Id: hal-01358916 https://hal.science/hal-01358916}

Submitted on 1 Sep 2016

HAL is a multi-disciplinary open access archive for the deposit and dissemination of scientific research documents, whether they are published or not. The documents may come from teaching and research institutions in France or abroad, or from public or private research centers.
L'archive ouverte pluridisciplinaire HAL, est destinée au dépôt et à la diffusion de documents scientifiques de niveau recherche, publiés ou non, émanant des établissements d'enseignement et de recherche français ou étrangers, des laboratoires publics ou privés. 


\title{
Multi-scale surface characterization in additive manufacturing using $\mathrm{CT}$
}

\author{
Yann QUINSAT $^{1^{*}}$, Claire LARTIGUE ${ }^{1}$, Christopher A. BROWN ${ }^{2}$, Lamine \\ HATTALI ${ }^{3}$
}

${ }^{1}$ LURPA, ENS Cachan, Univ. Paris-Sud, Université Paris Saclay, 94235 Cachan, France

${ }^{2}$ Surface Metrology Lab, WPI, USA

${ }^{3}$ Fast Univ. Paris-Sud, Université Paris Saclay 91405 Orsay, France

* Corresponding author. Tel.: +33 147402 213; fax: +33 147402 000. E-mail address:

yann.quinsat@lurpa.ens-cachan.fr

\begin{abstract}
In additive manufacturing, the part geometry, including its internal structure, can be optimized to answer functional requirements by optimizing process parameters. This can be performed by linking process parameters to the resulting manufactured geometry. This paper deals with an original method for surface geometry characterization of printed parts (using Fused Filament Fabrication FFF) based on 3D Computer Tomography (CT) measurements. From 3D measured data, surface extraction is performed, giving a set of skin voxels corresponding to the internal and external part surface. A multi-scale analysis method is proposed to analyse the relative internal area of the total surface obtained at different scales (from sub-voxel to super-voxel scales) with different process parameters. This analysis turns out to be relevant for filling strategy discrimination.
\end{abstract}

Keywords: CT measurement, additive manufacturing, multi-scale analysis

\section{Introduction}

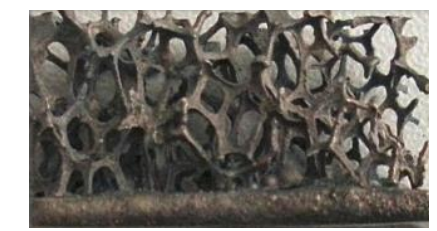

Fig. 1. Example of internal structure

In additive manufacturing, the weight of parts can be optimized to support a given load by designing an internal structure, including porosity, using a given filling strategy (figure 1). Relations between strength and filling strategies are required for product and process design optimization. Our preliminary study has 
shown the significant influence of FFF (Fused Filament Fabrication) process parameters on both the mechanical and fracture properties of the manufactured parts.

Within the context of fracture study, a series of bending samples has been carried out by considering variable filling parameters such as layer thickness, filling mode, and density. All the specimens presented a small cracking directly issued from manufacturing (figure 2(a)). Following cracking initiation, a 3-point bending test was performed with the view to determining $\mathrm{K}_{\mathrm{C}}$, critical stress intensity factor corresponding to fracture initiation. Results displayed in table 1 clearly highlight the great influence of the process parameters on the value of Kc. Therefore, the relations between strength and filling strategies are necessary for product and process design optimization.

Table 1. Values of Kc (from DOE define in table 3)

\begin{tabular}{llllllllll}
\hline Tests & 1 & 2 & 3 & 4 & 5 & 6 & 7 & 8 & 9 \\
\hline Kc (MPa.sqrt(m)) & 0.54 & 1.27 & 1.68 & 0.99 & 2.03 & 0.78 & 0.55 & 0.68 & 0.61 \\
\hline
\end{tabular}

In the paper, we propose a first approach to determine the relation between the filling strategy and the resulting surface topography, both internal and external, which can be linked to strength. Surface topography is thus considered the key element between the manufacturing process and the part function.

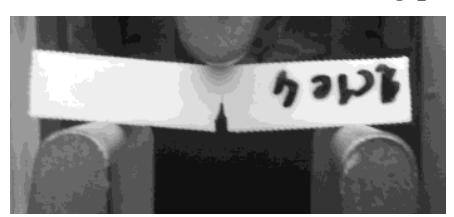

(a) 3-point bending setup

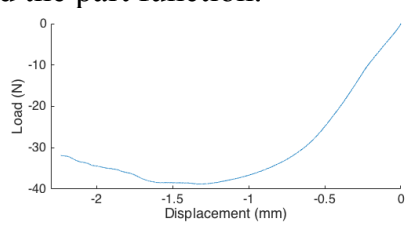

(b) Typical load evolution vs displacement

Fig. 2. Description of the cracking tests

The study of 3D surface topography obtained by FFF is a problem welladdressed in the literature. The most classical approximation consists in modeling the external surface as a juxtaposition of elliptical pipes (or tubes) [1]. The influence of scanning and deposition directions on external topography during filling has been studied in [2,3]. Zeng et al. show the influence of the process on local curvatures in a multi-scale analysis [4]. An approach based on a finite element analysis is proposed in [5] in order to predict 3D surface topography issued from FFF based on a few measured points. Meanwhile, Pupo et al. [6] establish the link between process parameters and the obtained topography in the case of sintering of metallic powders. Hence, most studies address the influence of process parameters on external surface topography, but few works have studied the effect of process parameters on porosity. One difficulty lies in measuring the internal part surface. Computer Tomography (CT) now allows non-destructive 3D measurements of internal and external part geometry with uncertainties down to micrometers $[7,8,9]$. 

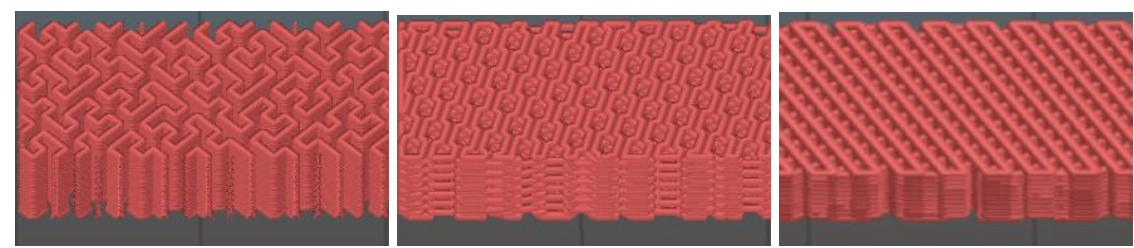

Fig. 3. The 3 different filling strategies: (a) Hilbert curve (b) Honey curve(c) ZigZag curve

Data issued from tomography represent the different levels of X-ray attenuation of the part for each point of the measured volume. The information is stored as a numerical value (gray level) in a volume element called voxel [10]. Surface extraction from these $3 \mathrm{D}$ data (also referred to as $3 \mathrm{D}$ edge detection) is the first necessary stage for metrology applications $[11,12]$, and is strongly linked to the voxel size which represents the tomography resolution [13]. Some studies show that uncertainty in surface extraction can be reduced by making use of a sub-voxel resolution method which numerically improves CT resolution [12, 10]. Uncertainty can achieve $1 / 10$ of the voxel size when sub-voxel resolution is used [14], which makes CT applied for dimensional metrology applications.

Within this context, this paper deals with a method for surface characterization in additive manufacturing to discriminate the filling strategy based on CT measurements. The objective is to propose a tool to discriminate the internal geometry based on the relative internal area evaluation. The originality of this approach lies in the use of a multi-scale discrimination method, which analyses the relative internal area of the total surface, including internal surfaces of pores from CT measurements. For the experimentation, parts were made using 3-D printer (Ultimaker 2) with three different filling strategies as displayed in figure 3. For each specimen, the same layer thickness ep $=0.25 \mathrm{~mm}$ was used. This paper is organized as follows. Section 2, is dedicated to the presentation of our method for CT data treatment. In section 3, the surface area, including that of the pores, is characterized as a function of scale. The method application is proposed in section 4 .

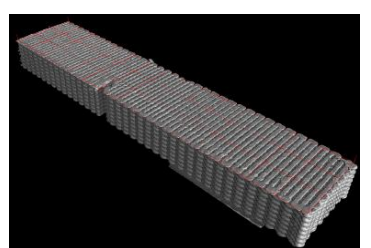

(a) Example of one measured part

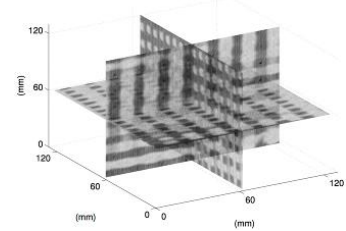

(b) Data representation

Fig. 4. Measurement from CT

\section{CT data treatment}

Data obtained by CT measurements correspond to a set of images defined in 3 perpendicular directions (see figure 4(b)) which constitute a set of voxels. For me- 
trology applications, the part geometry is defined from the contours of its surface (both external and internal). Ontiveros et al [15] propose a review of surface extraction methods in relation with metrology applications. Among all the methods, threshold methods are commonly used for industrial applications: a gray level value is defined as a limit to differentiate the air and the material [15].

To conduct a surface characterization by multi-scale analysis, an automatic CT data treatment method is required. Hence, a threshold method, close to the algorithm proposed in [17], and using Matlab@ Image Processing Toolbox, has been developed. The first step is then image binarizing according to a threshold value determined with the well-known Otsu's method [16]. Otsu's thresholding chooses the threshold to minimize the intraclass variance of the thresholded black and white pixels. This step is followed by a morphological operation on the binary image which removes interior pixels and keeps the outline of the shapes. As surface extraction is the key point of surface characterization, and the basis for the evaluation of metrological quantities, we propose to assess our method.

For this purpose, a gauge block made in $\mathrm{ZrO} 2$ is measured. This gauge ensures a flatness defect of $0.10 \mu \mathrm{m}$ and a parallelism defect between its two faces of 0.10 $\mu \mathrm{m}$. The calibrated distance between the 2 faces is $1.3 \mathrm{~mm}$ with an uncertainty of $\pm 0.12 \mu \mathrm{m}$ at $20^{\circ} \mathrm{C}$. CT measurements are performed with a Zeiss Metrotom for which the voxel size is $5.9 \mu \mathrm{m} \times 5.9 \mu \mathrm{m} \times 5.9 \mu \mathrm{m}$. Finally, the measured volume consists of $200 \times 300 \times 1132$ voxels. Our extraction method is applied leading to 2 sets of points, each one corresponding to one of the 2 gauge faces (figure 5). To test the interest of the sub-voxel technique, the extraction method is applied considering a smaller voxel-size, $30 \%$ of the original voxel-size. This is simply obtained by a sub-voxel linear interpolation.

Assessment is thus performed considering two quality indicators, as proposed in [18]: noise and trueness. Noise accounts for the measurement dispersion around each extracted face (a plane in practice), and trueness measures the difference between the calibrated distance and the measured distance obtained after face extraction (plane-plane distance). Results, displayed in table 2, are consistent with the gauge quality, which illustrates the efficiency of our surface extraction method. It also can be observed that the noise along with the trueness is decreased when using a smaller voxel-size. This interesting result shows that sub-pixel refinement, as suggested by numerous studies $[19,15,10]$ actually improves surface extraction.

Table 1. Assessment of the surface extraction method

\begin{tabular}{llll}
\hline Voxel-size & Noise $($ Face 1) $(\mu \mathrm{m})$ & Noise $($ Face 2) $(\mu \mathrm{m})$ & Trueness $(\mu \mathrm{m})$ \\
\hline original size & 3 & 3.3 & 25.5 \\
$30 \%$ of original size & 2.4 & 2.7 & 21.2 \\
\hline
\end{tabular}



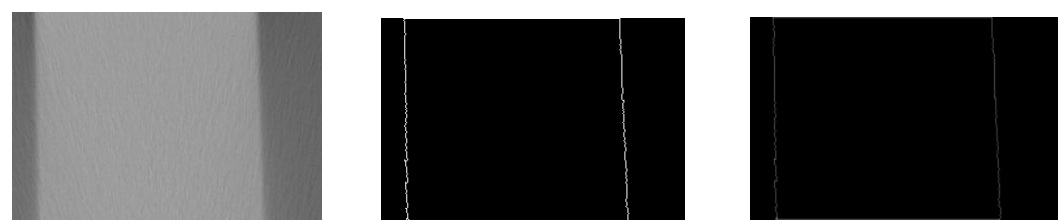

Fig. 5. Contour extraction for the gauge block: (left) Gauge Measurement, (middle) Contour extraction (original voxel-size) (right) Contour extraction ( $30 \%$ of the original voxel- size)

\section{Surface characterization by multi-scale analysis}

To link process parameters with the resulting surface, a multi-scale discrimination method is proposed to analyze the relative internal area of the total surface. The surface area, including that of the pores, is characterized as a function of scale. Prior to the description of our multi-scale analysis method, the calculation of the surface area based on the identification of the skin voxels is first detailed.

\subsection{Relative area calculation}

The area of the part surface can be defined from the 3D extracted contours, these contours defining a kind of skeleton structure. Hence, the skeleton is a $3 \mathrm{D}$ element. To simplify the representation, an example of the skeleton's construction in $2 \mathrm{D}$ is reported in figure 6.

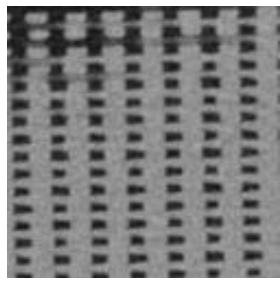

(a) Measurement

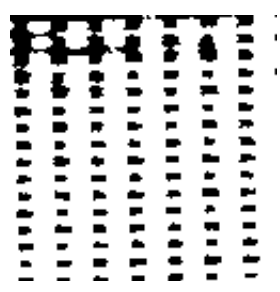

(b) Image binarizing

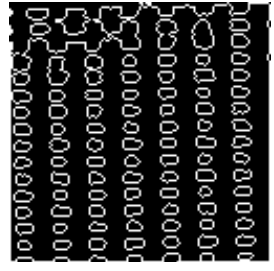

(c) Skeleton structure

Fig. 6. Surface extraction in the XY-plane

Indeed, the operation of surface extraction leads to the identification of all the voxels containing a portion of surface (internal or external) referred to as skin voxels, $\mathrm{V}_{\text {skin }}$ (figure $7(\mathrm{~b})$ ). The relative internal area is calculated as the sum of all the areas of the skin voxel faces:

$$
A=\sum_{i=V_{\text {Skin }}} \sum_{j=1}^{6} A_{i, j} \cdot \delta_{i, j}
$$


Where

$\left\{\begin{array}{l}\delta_{i, j}=1, \text { if thefacei belongingto the voxel } j \text { is in contactwith the air } \\ \delta_{i, j}=0, \text { if thefacei belongingto the voxel } j \text { is not in contactwith the air }\end{array}\right.$

The area so calculated depends on the dimensions of the studied zone. To avoid this problem, the relative internal area $A_{r}$ is introduced, defined by $A_{r}=A / A_{b}$, where $A_{b}$ is the area of the big voxel bounding the studied zone (figure 7(a)). Considering a $2 \mathrm{D}$ representation of a specimen measurement, the set of skin voxels corresponds to the white voxels in figure $7(\mathrm{c})$.

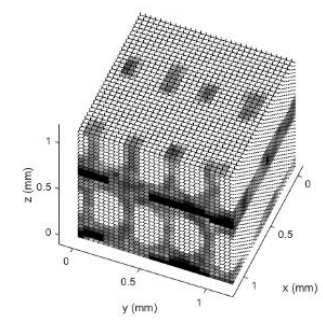

(a) Original voxel map

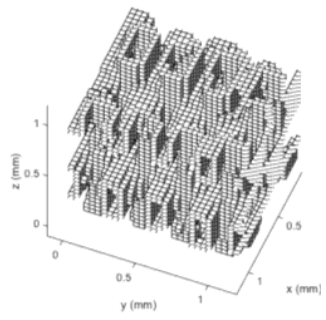

(b) Skin voxel representation

Fig. 7. Voxel representation of surface extraction

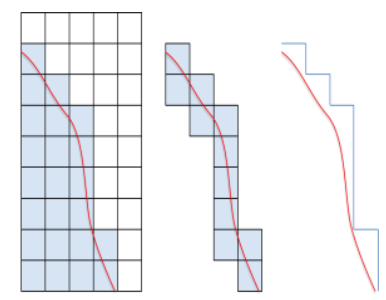

(c) Skin voxel extraction

\subsection{Multi-scale analysis}

In order to conduct a multi-scale analysis, the surface area is characterized as a function of scale [20]. The voxel size defines the scale, and we propose to vary this value to have scales smaller and larger than the original dimension related to the measurement scale. The value of the material density belonging to the considered voxel is obtained by linear interpolation. The influence of such a variation on the surface extraction is illustrated in figure 9.
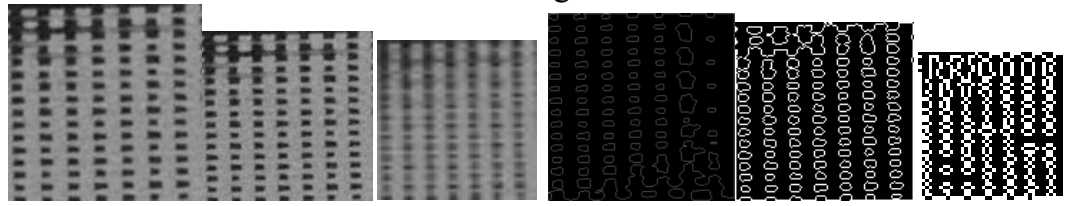

Fig. 9. Surface extraction as a function of scale (defining from initial voxel size) - from left to right: Measurement scale 0.3 , scale 1 , and scale 3 , Surface extraction scale 0.3 , scale 1 and scale 3

The parameter $A_{r}$ can thus be calculated as a function of scale. The scale is chosen as the area of the voxel surface for a considered voxel size. The presented method proposes to vary this voxel size (i.e. the scale) and to evaluate the corresponding internal surface area, the scale varying from sub-voxel to meta-voxel. The relative internal area evolves, increasing linearly on a decreasing logarithmic scale to a plateau (see figure 10). The plateau begins at the scale of the original measured 
voxel (identified by a vertical blue line in the figure). Results highlight that, in this study, the numerical sub-voxel treatment (scales $<10^{-2}$ in the present case) does not provide additional information. In the same way, meta-voxels are too far from reality. When the size of the voxel is close to the size of the test part, the relative internal area becomes lesser than 1, which is not realistic. The study can thus be restricted to the zone in which the relative internal area is above 1.

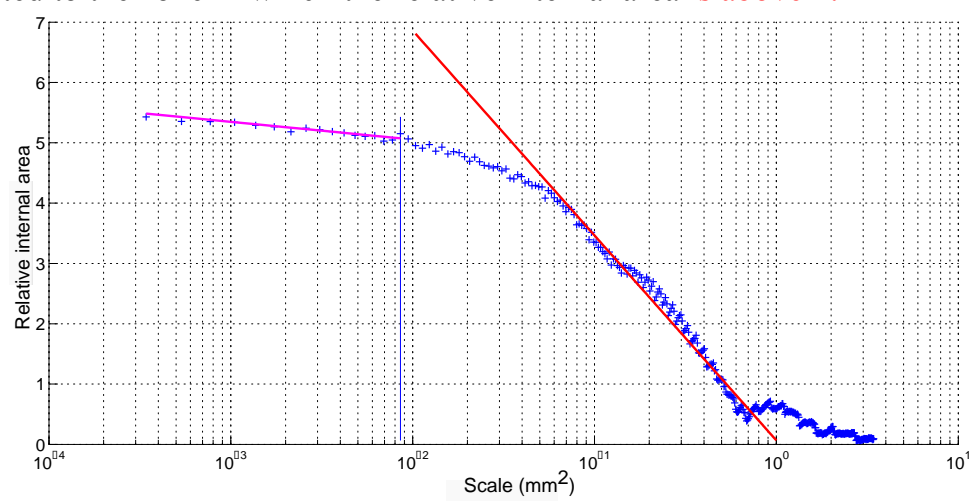

Fig. 10. Evolution of the relative internal area as a function of the scale

\section{Application and discussion}

The interest of our surface characterization method is illustrated through 2 applications. The first one concerns the use of such a method to discriminate filling modes, and the second one more particularly addresses the influence of process parameters on resulting printed surfaces.

\subsection{Filling mode discrimination}

For this application, 3 different filling modes are used as described in figure 3 with the same process parameters: thickness is set to $0.25 \mathrm{~mm}$, and the filling is imposed to $60 \%$. The original voxel sizes are $37.8 \mu \mathrm{m} \times 37.8 \mu \mathrm{m} \times 37.8 \mu \mathrm{m}$, and the bounding voxel size is set to $5 \mathrm{~mm} \times 5 \mathrm{~mm} \times 5 \mathrm{~mm}$. The multi-scale analysis is applied to the CT measurement obtained for the 3 specimens. Results, displayed in figure 11 , show that the evolution of the relative internal area is different depending on the filling mode. This is particularly distinct at low scales.

The linear decrease with the increase of the scale is in turn greater when the original relative internal area is the largest, corresponding to the zig-zag strategy. This analysis turns out to be relevant for filling strategy discrimination: multiscale analysis of the relative internal area enables printed surface characterization. 


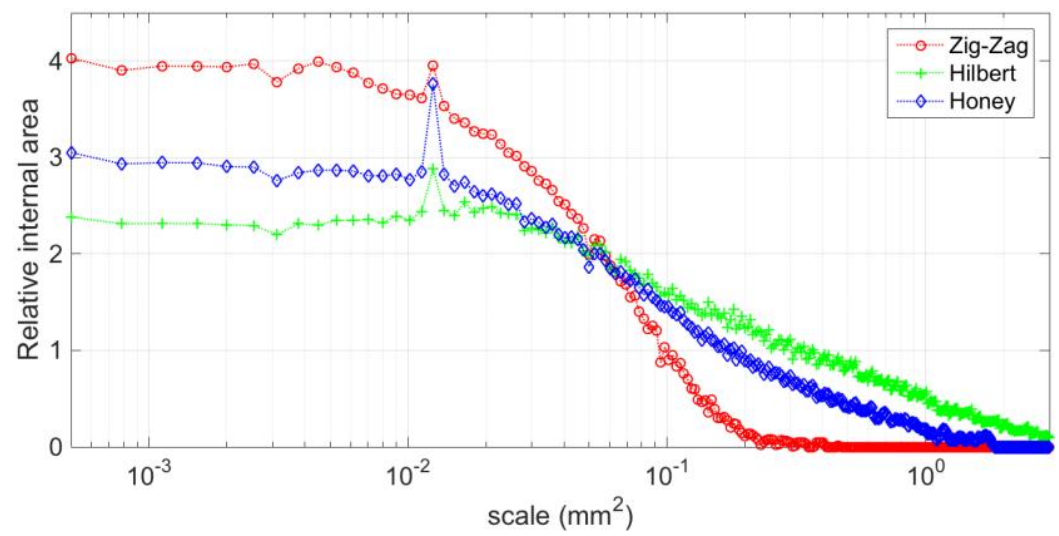

Fig. 11. Filling mode comparison using mutli-scale analysis

\subsection{Study of process parameters}

The second illustration aims at studying the influence of process parameters on the manufactured surface. The zig-zag mode is considered for this study. The process parameters retained are level thickness, direction of filling, and filling rate. A design of experiment is proposed according to a table L9 with 3 factors for 3 levels (table 3).

Table 3. Design of experiment.

\begin{tabular}{llll}
\hline Factor & Level1 & Level2 & Level3 \\
\hline Thickness $(\mathrm{mm})$ & 0.15 & 0.2 & 0.25 \\
Direction $\left({ }^{\circ}\right)$ & 0 & 30 & 45 \\
Filling rate $(\%)$ & 60 & 80 & 100 \\
\hline
\end{tabular}

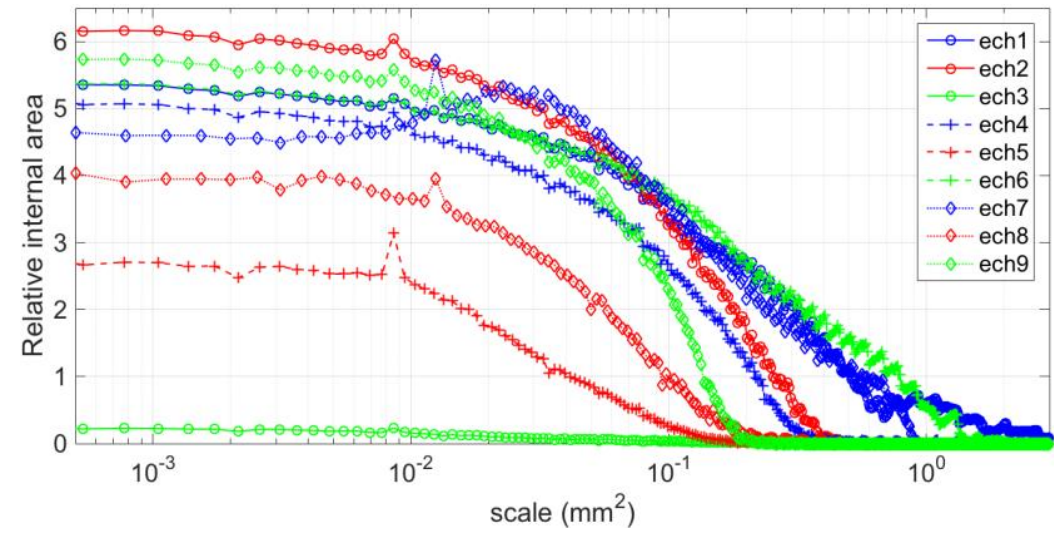

Fig. 12. Multi-scale analysis for different process parameters 
The 9 manufactured specimens are measured using CT and a multi-scale analysis of the relative internal area is afterwards performed, leading to the results reported in figure 12. As displayed in the figure, the value of the relative internal area at the low scales is different in function of the considered specimen. Furthermore, in the linear zone, the slope also varies for each specimen. This study confirms the relevance of using the relative internal area to discriminate surface topographies obtained using different process parameters. It is interesting to notice that due to process uncertainties, specimen for which the filling rate is $100 \%$ present some pores, which explains that the relative internal area is not constant for these specimens. This is likely due to process uncertainty.

\section{Conclusion}

The set of skin voxels defined the 3D surface topography, both internal and external. To link process parameters to the manufactured surface, a multi-scale discrimination method is proposed which analyses the relative internal area of the total surface. The surface area (area of all the skin voxels), including that of the pores, is characterized as a function of scale. To show the relevance of multi-scale relative internal area analysis, 2 different applications are proposed. The first one clearly highlights the interest of our approach to discriminate filling modes when using FFF. For the second application, the filling mode is the same (zig-zag mode), and the study focuses on the influence of some process parameters (level thickness, filling direction, and filling rate) on the printed surface. This study confirms the relevance of using the relative internal area to discriminate surface topographies obtained with different process parameters. Hence, the geometry and the structure of the part can be analyzed according to process parameters to answer a given part function. This work is a first approach to determine the relationship between mechanical properties and surface topography. We propose to use the relative internal surface area to discriminate the internal geometry. According to multiscale analysis, further work will focus on linking strength and internal relative area. A functional correlation could be found by regressing the relative internal areas at each scale versus the mechanical properties.

Acknowledgments Authors would thank Zeiss Industrial Metrology, LLC which provides all the CT measurements.

\section{References}

1. Ahn, D., Kweon, J.H., Kwon, S., Song, J., Lee, S.: Representation of surface roughness in fused deposition modeling. Journal of Materials Processing Technology 209(15-16), 5593 $5600(2009)$

2. Galantucci, L., Lavecchia, F., Percoco, G.: Experimental study aiming to enhance the surface finish of fused deposition modeled parts. CIRP Annals - Manufacturing Technology 58(1), $189-192(2009)$ 
3. Pandey, P.M., Reddy, N.V., Dhande, S.G.: Improvement of surface finish by staircase machining in fused deposition modeling. Journal of Materials Processing Technology 132(1-3), 323 $-331(2003)$

4. Zeng, Y., Wang, K., Wang, B., Brown, C.: Multi-scale evaluations of the roughness of surfaces made by additive manufacturing. In: ASPE - 2014 Spring Topical Meeting (2014)

5. Jamiolahmadi, S., Barari, A.: Surface topography of additive manufacturing parts using a finite difference approach. Journal of Manufacturing Science in Engineering 136(4), 1-8 (2014)

6. Yurivania, P., KarlaP, M., Joaquim, C.: Influence of process parameters on surface quality of cocrmo produced by selective laser melting. The International Journal of Advanced Manufacturing Technology 80(5-8), 985-995 (2015)

7. Wang, J., Leach, R.K., Jiang, X.: Review of the mathematical foundations of data fusion techniques in surface metrology. Surface Topography: Metrology and Properties 3(2), 023,001 (2015)

8. Chiffre, L.D., Carmignato, S., Kruth, J.P., Schmitt, R., Weckenmann, A.: Industrial applications of computed tomography. CIRP Annals - Manufacturing Technology 63(2), 655 - 677 (2014)

9. Bartscher, M., Hilpert, U., Goebbels, J., Weidemann, G.: Enhancement and proof of accuracy of industrial computed tomography (ct) measurements. CIRP Annals - Manufacturing Technology 56(1), 495 - 498 (2007)

10. Yage-Fabra, J., Ontiveros, S., Jimnez, R., Chitchian, S., Tosello, G., Carmignato, S.: A 3d edge detection technique for surface extraction in computed tomography for dimensional metrology applications. CIRP Annals - Manufacturing Technology 62(1), 531 - 534 (2013)

11. Dewulf, W., Kiekens, K., Tan, Y., Welkenhuyzen, F., Kruth, J.P.: Uncertainty determination and quantification for dimensional measurements with industrial computed tomography. CIRP Annals - Manufacturing Technology 62(1), 535 - 538 (2013)

12. Lifton, J.J., Malcolm, A.A., McBride, J.W.: On the uncertainty of surface determination in $\mathrm{x}-$ ray computed tomography for dimensional metrology. Measurement Science and Technology 26(3), 035,003 (2015)

13. Kruth, J., Bartscher, M., Carmignato, S., Schmitt, R., Chiffre, L.D., Weckenmann, A.: Computed tomography for dimensional metrology . CIRP Annals - Manufacturing Technology 60(2), $821-842(2011)$

14. Carmignato, S.: Accuracy of industrial computed tomography measurements: Experimental results from an international comparison. CIRP Annals - Manufacturing Technology 61(1), $491-494$ (2012)

15. Ontiveros, S., Yage, J., Jimnez, R., Brosed, F.:Computer tomography 3d edge detection comparative for metrology applications. Procedia Engineering 63, 710 - 719 (2013). The Manufacturing Engineering Society International Conference, MESIC 2013

16. Otsu, N.: A threshold selection method from gray-level histograms. IEEE Transactions on Systems, Man, and Cybernetics 9(1), 62-66 (1979)

17. Shahabi, H., Ratnam, M.: Simulation and measurement of surface roughness via grey scale image of tool in finish turning. Precision Engineering 43, pp.146 - 153(2016)

18. Mehdi-Souzani, C., Quinsat, Y., Lartigue, C., Bourdet, P.: A knowledge database of qualified digitizing systems for the selection of the best system according to the application. CIRP Journal of Manufacturing Science and Technology pp. - (2016)

19. Jiménez,R.,Comps,C.,Yague,J.: An optimized segmentation algorithm for the surface extraction in ncomputed tomography for metrology applications. Procedia Engineering 132, $804-$ 810 (2015). MESIC Manufacturing Engineering Society International Conference 2015

20. Brown, C.A., Johnsen, W.A., Hult, K.M.: Scale-sensitivity, fractal analysis and simulations. International Journal of Machine Tools and Manufacture 38(5-6), 633 - 637 (1998) 